\title{
Elementi metodologici per una riflessione sui dati dell'epidemia di SARS-CoV-2
}

\author{
Anteo Di Napoli ${ }^{1}$, Francesco Franco ${ }^{2}$, Giuseppe Quintaliani ${ }^{3}$ \\ ${ }^{1}$ Comitato Epidemiologia della Società Italiana di Nefrologia, Roma - Italia \\ ${ }^{2}$ Editorial Board, Giornale di Clinica Nefrologica e Dialisi, Epidemiologia e Statistica, Roma - Italia \\ ${ }^{3}$ Direttivo della Società Italiana di Nefrologia, Perugia - Italia
}

\begin{abstract}
Methodological elements for a reflection on the data of SARS-CoV-2 epidemic
Findings of the seroprevalence survey conducted by Istat between May 25 and July 15 2020, on a sample of 64,660 people, show that only $2.5 \%$ of Italian people developed antibodies to SARS-CoV-2, a prevalence very far from the hypothesis of achieving herd immunity. Starting from the comment on these results, we summarized some of the main indicators used to evaluate the epidemic curves $\left(R, R_{0}, R_{t}\right)$ and the concept of herd immunity. $R_{0}$, basic reproduction number, represents the average number of secondary cases we expect to observe from a single primary case in a population with no immunity to the disease before prevention and containment measures have been planned. $R_{t^{\prime}}$ effective reproduction number, is calculated over time and considers how the outbreak progresses, as a result of the containment measures and of people who might have gained immunity, because they survived from infection or were vaccinated. We presented the issue of herd immunity, or community immunity, a state of protection in a population obtained because the number of people in the population who are immune to infectious disease is above a critical threshold, resulting in a protection even for those who are not immune.
\end{abstract}

Keywords: Health policies, Herd immunity, R-reproduction number, SARS-CoV-2

\section{Introduzione: scenario epidemiologico}

Lo scorso 3 agosto, I'Istat ha reso pubblici i primi risultati dell'indagine di sieroprevalenza sul SARS-CoV-2, condotta tra il 25 maggio e il 15 luglio in contitolarità con il Ministero della Salute (1). Lo scopo dell'indagine era la stima della quota di persone nella popolazione generale che hanno sviluppato una risposta anticorpale contro SARS-CoV-2, attraverso la ricerca di anticorpi specifici nel siero. Per tale finalità non sarebbe stato adatto il solo tampone nasofaringeo, che identifica l'RNA virale solo in persone infette al momento del test.

I risultati dell'indagine si riferiscono a un campione di 64.660 persone. La prevalenza stimata (mediante metodi di

Received: August 18, 2020

Accepted: August 27, 2020

Published online: September 30, 2020

Indirizzo per la corrispondenza:

Anteo Di Napoli

Società Italiana di Nefrologia

Viale dell'Università 11

00185 Roma RM - Italia

anteo.dinapoli@libero.it riporto dei risultati campionari alla popolazione) di persone con IgG positivo, che hanno, cioè, sviluppato gli anticorpi per il SARS-CoV-2, è stata di 1.482 .000 persone (2,5\% della popolazione). Tale dato è 6 volte superiore a quello del numero di casi comunicati dall'Istituto Superiore di Sanità, identificati attraverso I'utilizzo dei tamponi per la ricerca dell'RNA virale. Tuttavia, un valore così basso di sieroprevalenza indica una corrispondente percentuale di suscettibili nel Paese del $97,5 \%$, rendendo inimmaginabile il raggiungimento della cosiddetta immunità di gregge. L'indagine ha confermato le forti differenze territoriali, con valori di sieroprevalenza che, in Lombardia, hanno raggiunto il $7,5 \%$, con un picco del $24 \%$ in provincia di Bergamo, dove, in val Seriana, la prevalenza è stata intorno al $60 \%$. Nel Mezzogiorno la sieroprevalenza è stata inferiore all' $1 \%$.

Interessante il dato sulla sieroprevalenza più elevata osservata tra i lavoratori della Sanità $(5,3 \%)$, rispetto a tutte le altre categorie professionali, con valori del 9,8\% nelle zone con maggiore sieroprevalenza complessiva. II dato, oltre a confermare l'elevato tributo pagato da medici e infermieri nella lotta al virus, sottolinea, tuttavia, come, probabilmente, il SSN si sia fatto trovare impreparato rispetto alla sfida, sia pure inedita e imprevedibile, rappresentata dal 
nuovo coronavirus. Non si è tenuto conto del "Piano nazionale di preparazione e risposta a una pandemia influenzale", elaborato dal Ministero della salute nel 2008, ma, in Lombardia, epicentro italiano della pandemia, "si è scambiata un'emergenza che era di sanità pubblica per un'emergenza di terapie intensive. All'inizio, non sono stati isolati i casi, non sono state fatte le indagini epidemiologiche, non sono stati fatti i tamponi ai pazienti e i medici sono andati in giro senza protezione individuale... e, soprattutto, hanno involontariamente diffuso il contagio", come denunciato dal presidente dell'Ordine dei Medici di Bergamo, Guido Marinoni. Più in generale, è parso evidente come il personale sanitario abbia perso, negli anni, la capacità di lavorare in un contesto di alto rischio infettivo (2).

L'andamento dell'epidemia ha indotto il Governo italiano ad attuare il cosiddetto "lockdown" a partire dall'11 marzo, imponendo la limitazione degli spostamenti e misure di distanziamento fisico. Tra la fine di marzo e l'inizio di aprile, l'epidemia ha fatto registrare i valori di picco: 21 marzo per i nuovi casi (6657), 27 marzo per i decessi (969), 3 aprile per i ricoverati in terapia intensiva (4068) per Covid-19, 4 aprile per i ricoverati con sintomi (9010) (3). II miglioramento delle curve epidemiche ha portato il Governo italiano a decidere la riapertura delle attività commerciali del Paese, parziale dal 4 maggio e totale dal 3 giugno. Sono rimaste le misure di "distanziamento fisico", dell'utilizzo delle mascherine nei luoghi chiusi e di quarantena per persone provenienti da Paesi extra-Schengen.

L'incremento di nuovi casi nell'ultimo periodo indica che la circolazione di SARS-CoV-2 è ancora rilevante e che, in condizioni sfavorevoli, può attivare focolai anche estesi. La situazione epidemiologica è fluida e impone un atteggiamento di estrema prudenza, anche in considerazione del fatto che la pandemia sta facendo registrare nuovi record nel numero di contagi e decessi in molti Paesi (Stati Uniti, Brasile e India su tutti) e che, in molti Paesi europei, soprattutto balcanici, fa temere che l'innesco di una seconda ondata possa coinvolgere anche l'Italia (4).

La diversità negli interventi politici di messa in sicurezza della popolazione è molto evidente quando prendiamo in considerazione Paesi vicini che mostrano differenze notevoli in merito alla diffusione dell'infezione. Le differenze abissali tra Stati Uniti e Canada oppure Svezia e Finlandia fanno capire come una pandemia non possa essere affrontata solo sul piano sanitario, ma come debba essere affrontata anche e, forse, soprattutto sul piano politico e organizzativo. Abbiamo assistito a politiche ondivaghe, se non contraddittorie, come nel caso del Regno Unito, dove si è deciso prima nessun "lockdown", poi "lockdown", poi riapertura e, poi, mascherina obbligatoria. Per non parlare degli Stati Uniti. "Siamo i peggiori, i numeri non mentono", ha affermato Anthony Fauci (5). Anche in Italia la differenza di approccio tra Lombardia e Veneto ha contribuito a determinare importanti differenze in termini di esiti di salute (6).

\section{L'utilizzo dell'indice R per il monitoraggio dell'epidemia: interpretazione e limiti}

Per monitorare tali situazioni, variegate e complesse, si ricorre ad alcuni parametri di riferimento. Nelle settimane di "lockdown" e, soprattutto, con l'avvio delle cosiddette "fasi due e tre", anche i non addetti ai lavori hanno familiarizzato con un indice, " $R$ ", espresso, a volte, come " $R_{0}$ " e, altre volte, come " $R_{t}$ ". Di cosa si tratta, come si interpreta, qual è l'utilità e quali sono i limiti?

$\mathrm{R}$ è un indice che misura la capacità di un agente patogeno di diffondersi in una popolazione, indicando quante persone in media sono state infettate a partire da un soggetto infetto durante il suo periodo di infettività. Tale indice si calcola come rapporto tra il numero di soggetti che sono stati contagiati e il numero di soggetti che hanno diffuso l'infezione. È una misura che, in genere, si calcola retrospettivamente, basandosi sui numeri dei casi di infezione e su quello dei decessi osservati, e assume un valore tanto più elevato, quanto più l'agente patogeno è contagioso $(7,8)$.

I modelli più utilizzati per il calcolo di R utilizzano una struttura compartimentale della popolazione, corrispondente alle situazioni in cui ci si può trovare nel corso dell'epidemia. II modello SIR prevede che si può essere suscettibili (S) all'infezione, aver contratto l'infezione (I) ed essere guariti dall'infezione, cioè rimossi avendo ottenuto l'immunità permanente (R); il modello SIRS prevede che l'immunità sia temporanea e, quindi, si torna a essere suscettibili all'infezione dopo un certo tempo. Nel modello SIRD si tiene conto anche dei deceduti a causa dell'infezione (D). Nel caso del modello SIR si assume che il tempo di incubazione dell'infezione sia zero, la probabilità di incontro sia uguale per tutti gli individui della popolazione, il contagio avvenga per contatto diretto e la probabilità di guarigione sia costante per unità di tempo. Sulla base di tali affermazioni, il numero dei nuovi contagiati e, quindi, di nuovi potenziali contagianti per unità di tempo sarà proporzionale al numero di contatti tra suscettibili e infetti, tenendo conto degli individui che guariscono nell'unità di tempo. Si rimanda a testi specifici per approfondimenti (9).

L'utilizzo dell'indice $R$ fu proposto dall'epidemiologo George Mac Donald nel 1952, per studiare un'epidemia di febbre malarica (10). Una variante di $R$ è $R_{0}$, indice che si riferisce al tempo 0 dell'epidemia, quando si assume che tutta la popolazione sia suscettibile all'infezione, perché mai venuta in contatto con un determinato patogeno, prima che siano messe in atto misure di contenimento e di contrasto dell'infezione $(7,10)$. È appunto la situazione all'inizio dell'epidemia provocata dal nuovo coronavirus SARS-CoV- 2 .

L'indice $\mathrm{R}_{0}$, anche detto "numero di riproduzione di base", equivale al numero medio di infezioni secondarie causate da ciascun individuo infetto in tale popolazione. Quando vale 1 , indica che ogni infetto arriva a contagiare solo un altro soggetto, pertanto l'epidemia resta costante come numero di soggetti infetti. Se $R_{0}$ è superiore a 1 , l'epidemia crescerà 
esponenzialmente con un parametro funzione del valore di $R_{0}$. Nel caso in cui $R_{0}$ sia minore di 1 , l'epidemia tenderà a esaurirsi a una velocità tanto maggiore quanto minore è il valore dell'indice, fino al totale esaurimento della capacità dell'agente patogeno di infettare $(7,10)$.

$\mathrm{Se}$, a un certo istante $\mathrm{t}$, l'indice $\mathrm{R}$ risulta essere pari a 0,50 , ciò non significa che, per essere contagiati, bisogna incontrare due persone malate, secondo un'errata interpretazione. Vuol dire che, per esempio, da un campione di 100 malati, 50 persone (in media) verranno contagiate. Infatti, talune persone positive al virus contageranno molte persone (a causa dei molti contatti con persone suscettibili), mentre altre ne contageranno di meno e altre ancora non infetteranno nessuno grazie alla loro adesione alle misure di contenimento della diffusione del virus.

Da sottolineare che $R_{0}$ non fornisce alcuna indicazione sulla severità della malattia. Infatti, una patologia con alta letalità avrà un basso valore di $R_{0}$, in quanto $i$ deceduti non hanno più la possibilità di trasmettere l'infezione (11).

$L^{\prime}$ 'indice $R_{0}$ varia a seconda dell'agente patogeno e dipende da una serie di fattori, come la modalità di trasmissione dell'infezione (per via aerea o per contatto diretto), la durata dell'infettività, la densità, la frequenza di contatti e lo stato di salute complessivo degli individui della popolazione (11).

Di particolare rilevanza sono la durata dell'infettività, che tiene conto del periodo di incubazione, l'intervallo di tempo tra contagio e insorgenza dei sintomi, durante il quale soggetti asintomatici possono contagiare altre persone, e il periodo di latenza, corrispondente al tempo necessario perché un individuo contagiato diventi, a sua volta, contagioso. Di conseguenza, per lo stesso agente patogeno il valore di $R_{0}$ può variare nel corso di differenti episodi epidemici (11).

Nella realtà, infatti, i decisori e gli operatori di sanità pubblica, più che al valore di $\mathrm{R}_{0}$ fanno riferimento a quello dell'indice $R_{t}$, che misura il tasso di contagiosità in un determinato momento, in seguito all'applicazione di misure per il contenimento dell'infezione. Pertanto, la misura del tasso di riproduzione, vale a dire il numero di soggetti contagiati da una persona infetta nell'unità di tempo, indica la potenziale trasmissibilità dell'agente patogeno nel contesto per effetto dei provvedimenti adottati $(8,10)$.

Tuttavia, il calcolo di $R_{t}$ presenta delle criticità, in quanto dipende da una serie di fattori a partire dal numero di contatti del caso primario nell'unità di tempo e dalla durata della sua fase di contagiosità: più persone incontra, più dura la sua contagiosità e maggiore sarà il numero dei potenziali contagiati; l'indisponibilità dell'informazione relativa alla data di esordio della sintomatologia non consente un calcolo corretto di $R_{t}$. Infatti, generalmente, è nota solo la data di accertamento virologico dell'infezione attraverso il tampone. Essendo nota l'eterogeneità nel Paese relativa alla distanza dall'inizio dei sintomi al momento dell'esecuzione del tampone, questo può rendere difficile valutare le diverse generazioni di contagi $(8,10)$.
Inoltre, per il calcolo di $\mathrm{R}_{\mathrm{t}^{\prime}}$ è altrettanto importante stimare la probabilità di trasmettere l'infezione per ciascun contatto del caso primario, sulla quale fattori come il distanziamento fisico e l'utilizzo della mascherina hanno un ruolo molto importante. Va, poi, considerato che le probabilità di contagio differiscono a seconda della prossimità con un caso: i conviventi di un una persona in isolamento, in famiglia o in strutture residenziali, hanno maggiori probabilità di infezione (8).

Uno dei principali limiti di $R_{t}$ è che rappresenta la media dei casi nella popolazione di una certa area geografica; se si tratta di un intero Paese o di una grande regione, $R_{t}$ può non essere in grado di far emergere eventuali importanti cluster di infezione. Viceversa, in una piccola regione o in un'area più piccola, la presenza di un cluster anche piccolo può determinare un'impennata del valore di $R_{t}(7)$.

Per esempio, nelle scorse settimane, abbiamo osservato incrementi notevoli di $R_{t}$ in coincidenza con focolai di infezione, in alcune regioni (Molise, Umbria), poco popolose e con bassa prevalenza di infezione da SARS-CoV-2 (3).

Pertanto, l'indice $R_{t}$, valutato senza tenere conto del contesto in cui si è prodotto, può fuorviare la lettura del quadro epidemiologico da parte dei decisori politici e di sanità pubblica.

Una domanda che ricorre negli ultimi giorni è quella relativa all'osservazione, che, a fronte dell'incremento dei casi di infezione, non si registri un corrispondente aumento di ricoveri e decessi. Scrive il Prof. Bucci (Temple University di Filadelfia), sulla sua pagina Facebook: "A essere cambiati sono i pazienti, nel senso che cerchiamo, oggi, anche gli asintomatici, e la loro età mediana è passata da oltre 60 anni a 39 (dati ISS). Quindi, una semplice variazione nella distribuzione per età dei positivi: un classico effetto di popolazione (degli infetti, in questo caso). Inoltre, abbiamo cambiato il nostro comportamento e le persone più anziane sono più protette (sono più attente); i giovani, invece, si infettano percentualmente di più che in passato" (12).

\section{L'immunità di gregge nel contesto dell'epidemia da SARS-CoV-2}

Per chiudere questa sommaria rassegna, è necessario fare cenno alla questione della cosiddetta immunità di gregge, un meccanismo che si instaura all'interno di una comunità per cui, se la grande maggioranza degli individui acquisisce (naturalmente o attraverso un vaccino) l'immunità all'infezione, è protetto anche il resto della popolazione non immune alla malattia (11).

Ma qual è la soglia critica per raggiungere l'immunità di gregge o immunità di comunità? Dipende dalla malattia. Come si calcola? A partire da $\mathrm{R}_{0}$, indice su cui ci si basa per i programmi estesi di vaccinazione dei bambini. La quota di immuni da raggiungere e mantenere è calcolabile con la formula [1-(1/R)] ed è il criterio di valutazione dell'adeguatezza della proporzione di vaccinati raggiunta (8). 
Nel presentare l'indice $R_{0}$ si è premesso che ogni individuo della popolazione sia suscettibile all'infezione, affermazione che può valere solo al tempo zero dell'epidemia, in quanto $\mathrm{i}$ soggetti che si infettano e superano la malattia acquisiscono un'immunità che può essere temporanea o permanente.

Pertanto, nel tempo in cui l'epidemia si sviluppa, l'indice $R_{t}$ dipenderà dal valore di $R_{0}$ per la proporzione $(S)$ di individui nella popolazione ancora suscettibili: $R_{t}=R_{0}{ }^{*} S$. Più il numero di suscettibili diminuisce, per il corrispondente aumento di soggetti divenuti immuni, più la malattia avrà difficoltà a diffondersi. Per esempio, se $R_{0}$ fosse 2 , ma solo metà della popolazione fosse ancora suscettibile, il valore di $R_{t}$ scenderebbe a 1 (11).

La proporzione di soggetti immuni è il complemento 1-S di quella dei suscettibili; essendo $S=R_{t} / R_{0}$, per sostituzione la proporzione di immuni sarà: $1-\left(R_{t} / R_{0}\right)$.

Per tutti i valori di $R_{t}<1$, l'infezione tenderà a estinguersi nel tempo, e questo perché, nelle successive generazioni di infetti, il numero di casi sarà sempre minore. Pertanto, il valore di soglia critica $H$ (herd, gregge) per il raggiungimento dell'immunità di gregge, ipotizzando il caso di una popolazione completamente suscettibile, sarà $H=1-\left(1 / R_{0}\right)(11)$. Tale proporzione corrisponde alla quota di popolazione $V$ che deve essere vaccinata per raggiungere la soglia di immunità di gregge attraverso una vaccinazione, effettuata in modo random in una popolazione omogenea e totalmente suscettibile: $V=1-\left(1 / R_{0}\right)(13)$.

Nel caso del Covid-19, si è molto discusso di quale sia la soglia per il raggiungimento dell'immunità di gregge. II valore di $R_{0}$ stimato per l'infezione da SARS-CoV-2 è di 2,5. Accettando tale ipotesi, il valore critico sarebbe pari a: $[1-(1 / 2,5)]=0,6$, vale a dire che, se il $60 \%$ della popolazione raggiungesse l'immunità, l'epidemia sarebbe sotto controllo $(8,11)$.

La sieroprevalenza stimata delle persone che, in Italia, hanno contratto l'infezione da SARS-CoV-2 è stata, come detto, del 2,5\%, lontanissima dalla soglia critica ipotizzata e con un prezzo altissimo in vite umane già pagato al Covid-19 (35.000 morti). Tali dati indicano come sostanzialmente inaccettabile l'ipotesi di fermare l'epidemia attraverso il raggiungimento naturale dell'immunità di gregge. La sola alternativa praticabile per abbattere, efficacemente e rapidamente, la quota di popolazione suscettibile all'infezione e, quindi, spegnere l'epidemia appare essere quella della vaccinazione.

\section{Conclusioni}

In attesa del vaccino, è fondamentale proseguire con le misure imperniate sulle cosiddette " $3 T$ " (tracing, testing, treating), basate su identificazione precoce, trattamento, isolamento e sorveglianza attiva, che consentono sia di controllare potenziali catene di trasmissione sia di ridurre l'intervallo di tempo tra l'insorgenza dei sintomi e la diagnosi, il che ha contribuito a limitare la quota di casi che necessitano di ricovero ospedaliero, in particolare in terapia intensiva.
Un ausilio all'attività di tracciamento potrebbe derivare dall'utilizzo dell'applicazione "Immuni", che segnala se si è stati vicini a una persona risultata positiva al tampone (nell'ipotesi che entrambi abbiano scaricato l'applicazione). È stato dimostrato che tali ausili tecnologici, semplificando la ricostruzione della catena dei contagi, assicurano un beneficio in termini di salute pubblica, grazie alla più veloce identificazione dei casi e al conseguente più efficace e tempestivo contenimento della diffusione dell'infezione (14). "Immuni" è stata, tuttavia, scaricata solo da una piccola quota della popolazione, vanificando, per ora, tali potenziali benefici connessi all'applicazione.

In conclusione, facciamo nostro il messaggio del Prof. Remuzzi, Direttore dell'Istituto Mario Negri, che, a inizio luglio, diceva: "Se volete, possiamo chiudere la questione così, dicendo che, se oggi in Italia le cose vanno bene, ed è vero, è anche grazie al distanziamento sociale, all'uso delle mascherine e all'abitudine acquisita di lavarsi correttamente le mani. Che vanno mantenuti" (15).

\section{Bibliografia}

1. Istat. Primi risultati dell'indagine di sieroprevalenza sul SARSCoV-2. Roma, 3 agosto 2020. Online (Data di accesso 10/8/2020).

2. Curtale F. C'era una volta il piano pandemico. Salute Internazionale 2020. Online (Data di accesso 10/8/2020).

3. Ministero della salute. Covid-19 - Situazione in Italia. Online (Data di accesso 10/8/2020).

4. INMP. Indicazioni operative ad interim per la gestione di persone a elevata fragilità e marginalità socio-sanitaria nel quadro dell'epidemia di Covid-19. Roma: 2020. ISBN: 9788898544240. Online (Data di accesso 10/8/2020).

5. Adam D. A guide to R - the pandemic's misunderstood metric. Nature. 2020;583(7816):346-8. CrossRef

6. Salmaso $S . R_{0}$, un mito da superare. Scienza in Rete 2020. Online (Data di accesso 10/8/2020).

7. Cislaghi C, blog di. Come far di conto in un'epidemia. Epidemiologia \& Prevenzione 2020. Online (Data di accesso 10/8/2020).

8. Cislaghi $C$, blog di. $R_{0}, R_{t}, R_{t}$ che sono e che significano? Epidemiologia \& Prevenzione 2020. Online (Data di accesso 10/8/2020)

9. Nirenberg D. Herd immunity: How does it work? Medium. 2019 Dec 19. Online (Data di accesso 10/8/2020).

10. HuffPost. Fauci contraddice ancora Trump: "Siamo i peggiori, i numeri non mentono". HuffPost Italia, 6 agosto 2020. Online (Data di accesso 10/8/2020).

11. Lavezzo E, Franchin E, Ciavarella C, et al. Suppression of a SARSCoV-2 outbreak in the Italian municipality of Vo'. Nature. 2020;10. 1038/s41586-020-2488-1. CrossRef

12. Bucci E. Perché abbiamo meno morti. Facebook, 9 agosto 2020. Online (Data di accesso 10/8/2020).

13. Bartolozzi G. Vaccini e Vaccinazioni (Italian Edition). Edra Versione Kindle;271.

14. Kretzschmar ME, Rozhnova G, Bootsma MCJ, van Boven $M$, van de Wijgert JHHM, Bonten MJM. Impact of delays on effectiveness of contact tracing strategies for Covid-19: a modelling study. Lancet Public Health. 2020 July 16;5:e452-9. CrossRef

15. Cicchetti E. Le nostre armi contro il Covid e la guerra (fake) tra esperti. Parla Remuzzi. II Foglio, 3 luglio 2020. Online (Data di accesso 10/8/2020). 